\title{
Harald Høffding and the Historical Roots of the Bohrian Concept of Symbol
}

[Harald Høffding e as Raízes Históricas do Conceito Bohriano de Símbolo]

\author{
Hernán Pringe
}

\begin{abstract}
In this paper we analyze the historical roots of the Bohrian concept of symbol. More precisely, we argue that Bohr takes Kantian elements from Høffding's philosophy in order to develop his own concept of symbol. For this purpose, firstly, we focus on the two different senses that Bohr gives to the concept of symbol. Then, we study how each of these senses is related to different aspects of Høffding's philosophy and we show the connection between the Bohrian and the Kantian concept of symbol by means of Høffding's mediation.
\end{abstract}

Keywords: Analogy. Niels Bohr. Harald Høffding. Symbol.

Resumo: Neste artigo analizamos as raízes históricas do conceito bohriano de símbolo. Mais precisamente, afirmamos que Bohr toma elementos kantianos da filosofia de Høffding com a intenção de desenvolver seu próprio conceito de símbolo. Para esse propósito, primeiramente, nos focamos nos dois sentidos diferentes que Bohr oferece ao conceito de símbolo. Depois, estudamos como cada um desses sentidos está relacionado a diferentes aspectos da filosofia de Høffding e mostramos a conexão entre o conceito bohriano e o kantiano de símbolo por meio da mediação de Høffding.

Palavras-chave: Analogia. Niels Bohr. Harald Høffding. Símbolo.

\section{Introduction}

The aim of this paper is to investigate the historical connection between the Bohrian and the Kantian use of the concept of symbol.1 $!^{1}$ More precisely, we will try to show how the philosophy of Harald Høffding, Bohr's lifelong teacher and friend, provides Bohr with the
Kantian framework that enables him to cope with the epistemological problem posed by quantum theory. For this purpose, firstly, we will focus on the two different senses that Bohr gives to the concept of symbol (1.) Then, we will study how each of these senses is related to different aspects of Høff-

${ }^{*}$ PhD (2006), Universität Dortmund, Germany. Researcher at CONICET, Institute of Philosophy, University of Buenos Aires (Argentina) and Full Professor at the Institute of Philosophy, Universidad Diego Portales (Santiago, Chile). E-mail: hernan.pringe@mail.udp.cl. ORCID: https://orcid.org/0000-0001-7005-4838.

1 This paper is a modified version of Pringe (2007), pp. $124-139$. 
ding's philosophy (2., 3.) and we will show the connection between the Bohrian and the Kantian concept of symbol by means of Høffding's mediation.

\section{Bohr on Symbolic Knowledge}

Two senses may be distinguished in which Bohr considers our knowledge to be symbolic. We will call them the broad and the strict sense.2 In the first place, science in general and physics in particular provide us with symbolic knowledge because scientific concepts do not portray or depict reality. Their function is rather to bring about order among appearances, connecting them in a unified experience as extended and interconnected as possible. ${ }^{3}$ The purpose of science "is not to disclose the real essence of the phenomena but only to track down, so far as it is possible, relations between the manifold aspects of experience."(Bohr, 1934, p.18) This is particularly the case of physics. Physics does not study "something a priori given," since physics is just a method "for ordering and surveying human experience."(BOHR, 1963, p.10)

Quantum and classical physics are symbolic in this broad sense. In fact, Kramers recalls that "Bohr has expressed himself in discussions somewhat as follows: classical physics and the quan- tum theory, taken as descriptions of nature, are both caricatures." (HONNER, 1987, p. 158)

In the second place, there is another sense in which physics may be considered symbolic knowledge, but this meaning applies only to quantum and not to classical physics. In this second sense, symbolic knowledge is the cognition of what cannot be directly exhibited in intuition. Since atomic objects and processes cannot simultaneously satisfy the demands of spatio-temporal coordination and the claim of causality, atomic objects and processes cannot be directly exhibited in intuition and their knowledge is thus symbolic. Classical physics is not symbolic knowledge in this restricted sense.

In this connection, an epistolary exchange between Bohr and Christian Møller is particularly relevant. Møller asks Bohr explicitly about the meaning of the word "symbolic":

"The question at stake is what
one really understands under
the word 'symbolic' -what
does it mean that, e.g., the re-
presentation of a free particle
by means of de Broglie waves
is only a symbolic representa-
tion?" (STOLZENBURG, 1977,
p. 255)

"The question at stake is what one really understands under the word symbolic -what presentation of a free particle by means of de Broglie waves is only a symbolic representap. 255)

\footnotetext{
2 A third sense of symbol may also be distinguished, according to which Bohr uses this word as a synonym of "emblem." See Honner (1987), p. 154-155. But this sense has no relevance for our investigation.

3 "The task of science is both to extend the range of our experience and to reduce it to order" Bohr (1934, p. 1).
} 
But even more important to our discussion is that Møller, after acknowledging that all the signs we use to describe nature are symbols, poses the crucial question:

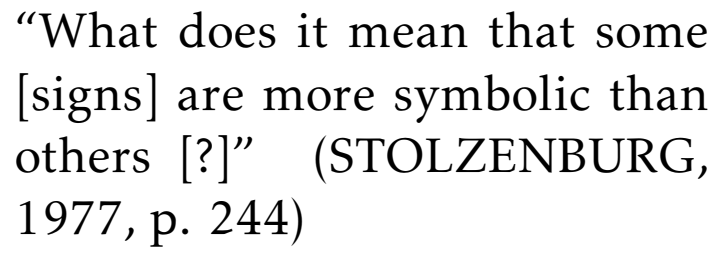

"What does it mean that some [signs] are more symbolic than others [?]" (STOLZENBURG, 1977, p. 244)

In other words, he demands for an explanation of the specific symbolic character of quantum theory, that distinguishes it from classical physics.

In his response, Bohr agrees that both classical and quantum physics make use of symbols. However, he maintains that there is a difference between both symbolisms and that this difference lies in their relation to intuition:

"I have only tried to underline the fact that in quantum theory we extensively use the same symbols as in classical theory, what nevertheless does not allow us to overlook the big difference between these theories and makes necessary great caution in the application of the forms of intuition to which the classical symbols are linked" (STOLZENBURG, 1977, p. $245-246) .4$

The quantum-mechanical formalism is symbolic because its concepts cannot be represented directly in intuition. This is so even in the case of the wave formalism, where the intuitive elements of the theory may hide the specific symbolic character that distinguishes it from any classical theory:

"If one considers the wave theory, its 'intuitiveness' is, however, precisely its strength and its trap at the same time and, by underlying the symbolic character of the approach, I have tried to point out here the great difference from the classical theories due to the quantum postulate, [a difference] that is not always taken sufficiently into account" (STOLZENBURG, 1977, p.245-246).

In the case of matrix mechanics, to the contrary, there is no doubt about the strict symbolic character of the formalism:

"Of course, it is not so easy to run into this danger in the matrix formulation, where rules that differ so much from

\footnotetext{
${ }^{4}$ Even though the "old quantum theory" should be distinguished from "quantum mechanics," Bohr still uses the expression "quantum theory" after the establishment of quantum mechanics, as it is clear in this passage. For Bohr, wave and matrix mechanics are just two different formulations of the same (quantum) theory.
} 
our usual algebra always make evident to us the peculiar essence of quantum theory; to use the word 'symbolic' for noncommutative algebra is by the way a language usage that goes back much further than quantum theory and it is incorporated in the general mathematical terminology" (STOLZENBURG, 1977, p.245-246).

As a matter of fact, Bohr underlines the peculiar symbolic character of quantum physics by putting quantum symbols in opposition to classical concepts. The latter can be exhibited in spatio-temporal pictures, while the former cannot.5

But to this sharp distinction between classical and quantum physics, a positive determination of their relationship follows: classical concepts symbolize atomic objects and processes. More concretely, it is by means of the concepts of isolated particle and free radiation that we exhibit quantum processes indirectly in intuition.6 These concepts are classical, since "such concepts as corpuscle and wave are only well defined within the scope of clas- sical physics, where, of course, light and electrons are electromagnetic waves and material corpuscles respectively."(BOHR, 1932, p.48) For this reason, "phrases such as 'the corpuscular nature of light' or 'the wave nature of electrons' are ambiguous."(BOHR, 1932 , p.48) This means that we are thereby not claiming that, e.g., electrons are waves, but just that they behave as if they were waves..$^{7}$ We establish a certain analogy between the behaviour of atomic objects and classical objects, which enables us to obtain an intuitive albeit indirect representation of the atomic entities.

In order to thoroughly account for the empirical evidence it is necessary to use incompatible classical images to represent atomic objects. For example, electrons behave in some situations as if they were waves, but in some others as if they were corpuscles. These incompatible images, each of which is indispensable for a complete account of the experiments, are called complementary representations. The extension and limits of their valid use is determined by the uncertainty relations set up by Heisenberg. Bohr considers these relations "as a direct expression of the absolute

\footnotetext{
${ }^{5}$ This is particularly stressed by Chevalley (1994, p. $\left.35 \mathrm{ff}\right)$.

6 "Hitherto we have only regarded certain general features of the quantum problema. This situation implies, however, that the main stress has to be laid on the formulation of the laws governing the interaction between the objects which we symbolize by the abstractions of isolated particles and radiation." (BOHR, 1934, p. 69).

7 "The extreme fertility of wave pictures in accounting for the behaviour of electrons must, however, not make us forget that there is no question of a complete analogy with ordinary wave propagation in material media or with non-substantial energy transmission in electromagnetic waves. Just as in the case of radiation quanta, often called 'photons,' we have here to do with symbols helpful in the formulation of the probability laws governing the occurrence of the elementary processes which cannot be further analysed in terms of classical physical ideas" (BOHR, 1932, p.48).
} 
limitation of the applicability of visualizable conceptions in the description of nature."(BOHR, 1934, p.114) Their specific task "consists in assuring quantitatively the logical compatibility of apparently contradictory laws which appear when we use two different experimental arrangements."(BOHR, 1937, p.293) Bohr

Heisenberg summarizes the situation as follows:

"[F]or visualization [of atomic processes] [...] we must content ourselves with two incomplete analogies -the wave picture and the corpuscular picture. The simultaneous applicability of both pictures is thus a natural criterion to determine how far each analogy may be "pushed" and forms an obvious starting-point for the critique of the concepts which have entered atomic theories in the course of their development, for, obviously, uncritical deduction of consequences from both will lead to contradictions. In this way one obtains the limitations of the concept of a particle by considering the concept of a wave. As N. Bohr has shown, this is the basis of a very simple derivation of the uncertainty relations between co-ordinate and momentum of a particle. In the same manner one may derive the limitations of the concept of a wave by comparison with the concept of a particle." 8

Briefly, quantum and classical physics provide us with symbolic knowledge because none of them is meant to copy an absolute reality. Their task as scientific theories is rather, using a Kantian expression, to "spell out appearances, so that they can be read as experience." 19 But the way in which this task is carried out by each of them is not the same. In classical physics, it is possible to exhibit concepts directly in intuition in order to gain a single and allencompassing image of nature. To the contrary, in quantum physics, such a single image cannot be obtained. Complementary images should be used so that atomic objects and processes are indirectly exhibited in intuition. This is the specific symbolic character of quantum physics Møller was asking about.

In the following, we will see how each of the two senses of the Bohrian concept of symbol is related to different aspects of Høffding's philosophy and that the connection between the Bohrian and the Kantian concept of symbol can therefore be established by means of Høffding's mediation.

\footnotetext{
${ }^{8}$ See: Heisenberg $(1949$, p. 11$)$

${ }^{9}$ Prol, AA 4: 312. Kant (2002, p.105-106).
} 


\section{Symbol in the Broad Sense}

In Høffding, the notion of symbol in its broad sense bears a close connection to the notion of analogy. ${ }^{10}$ According to Høffding, an analogy is the similarity of the relations between objects or parts of objects that may be very different in other respect. 11

Following Kant, ${ }^{12}$ Høffding distinguishes between quantitative and qualitative analogies. Quantitative analogies are called proportions. The relations that take part in a proportion are not merely similar, but identical. In this case, analogies are constructive, because one of the elements of the proportion can always be found by means of the other. A typical example of this kind of analogy is the relation between two numbers being identical to the relation between two other numbers. (HØFFDING, 1905, p.202)

On the contrary, qualitative analogies enable us to establish a priori only the relation between the unknown and something given, by means of another known relation. Analogies of this sort do not possess a constructive character, because the members that take part in the analogy have different origins. The main example of qualitative analogies is that between numerical and spatiotemporal series. At this point, Høffding follows Maxwell, who asserts:

"[A]ll the mathematical sciences are founded on relations between physical laws and laws of numbers, so that the aim of exact science is to reduce the problems of nature to the determination of quantities by operation with numbers."(MAXWELL, 1890, p. 156)

If an analogy between the numerical and the spatio-temporal series is established, it is possible through the former to determine a priori the place of the elements of the latter, although not their existence.13

However, such an analogy is in turn grounded by the analogy holding between the reason-consequence and cause-effect relations. In this regard, gHøffding affirms that the greatest merit of Kant's discussion of the a priori principles of the analogies of experience is to have rejected the dogmatic identification of reason and cause. (HØFFDING, 1905, p.203; 1923, p.72)

\footnotetext{
10 On the influence of Høffding on Bohr's thought, see especially Faye (1991) and Favrhold (1992).

11 “Vorläufig kann Analogie als Verhältnissähnlichkeit zwischen zwei Gegenständen definiert werden, eine Ähnlichkeit also, die sich nicht auf einzelne Eigenschaften oder Teile dieser Gegenstände, sondern auf das gegenseitige Verhältnis zwischen Eigenschaften oder Teilen gründet." (Høffding, 1923, p.1). Høffding defines analogy as the similarity and not the identity of relations because he considers identity in general as a certain degree or type of similarity: (1911, p. 193).

${ }^{12}$ A $179-180 /$ B 222.

13 Moreover, this analogy holds in the first place between different sciences, i.e., Mathematics and Physics, and it enables the applicability of the former to the latter. (HØFFDING, 1905b, p.99ff)
} 
Rather, the reason-consequence and the cause-effect relations stand to each other in a qualitative analogy. The former relation is a logical one between thoughts, the series of which is a work of our own spontaneity. To the contrary, the latter relation is a temporal one between events, the series of which is not produced by us but must be given to us. Although, according to Kant, one and the same function of synthesis establishes the formal ground-consequence and the temporal cause-effect relations, the necessity of the schematism of the categories for the formal relation to be applied to events implies the impossibility of a direct identification of them. ${ }^{14}$

This essential distinction between both kinds of series has for Høffding important consequences. On the one hand, only insofar as the series of empirical data and the formal series are analogous and not identical may we evade the danger of absolute idealism: 15

"The laws of thought may enable us to find our way through the world of experience; but from this we have no right to conclude that existence is estimated or in its fullness expressed in the laws of thought."(HØFFDING, 1905,

$$
\text { p.204) }
$$

On the other hand, a naive empiricism, which may argue that our concepts resemble in some way the empirical data, will also be untenable. According to Høffding, it is sufficient for the validity of thought that the relations between the members of the series of thoughts agree with the relations of the appearances. In this connection, Høffding adopts the ideas put forward by Hertz in his Principles of Mechanics: ${ }^{16}$

"We form for ourselves images or symbols of external objects; and the form which we give them is such that the necessary consequents of the images in thought are always the images of the necessary consequents in nature of the things pictured. [...]

The images we here speak of are our conceptions of things. With the thingsthemselves they are in conformity in one important respect, namely in satisfying the above-mentioned requirement. For our purposes it is not necessary that they should be in conformity with the things

\footnotetext{
14 Høffding nevertheless critizes Kant for not seeing that the concept of experience, which is grounded by means of analogies between formal and real series, is only an ideal that never looses its hypothetical character: (1923, p.76).

${ }^{15}$ Hegel and the Neo-Kantians of the Marburg's school are for Høffding examples of such idealism (HØFFDING, 1923, p.78 - 79).

16 For Høffding's reading of Hertz, see Høffding (1905b, p.110ff). See also Christiansen (2006).

17 In turn, Hertz's ideas are intimately connected with Helmholtz's doctrine of symbols. We may here indicate that, according to Helmholtz, "our sensations are indeed effects produced in our organs by external causes; and how such an effect expresses itself
} 
in any other respect."(HERTZ, 1956, p. 1-2 ${ }^{17}$

Høffding rejects the dogmatic conception of truth, which supposes a given, absolute essence of things that is to be reproduced by thought. In Høffding's opinion, dogmatism leads to scepticism, since the latter accepts this definition of truth, but affirms that this notion remains illusory. To both dogmatism and scepticism, Høffding opposes the critical conception of truth, according to which objective validity consists in the lawful connection of phenomena. The aim of thought is only to bring about order among phenomena, and this can be done only if our representations bear an inner connection such that the connection of the phenomena can be expressed and foretold by it.

For this purpose, no resemblance between our representations and the phenomena is required. No identity, but only an analogy between thought and phenomena, needs to be esta- blished. Moreover, as we have seen, only such an analogy can in fact be established, because we do not produce, through our thought, the series of phenomena in its existence. In this way, Høffding opposes the notion of symbolic knowledge, based on an analogy between thought and phenomena, to the notion of resembling knowledge, based on an identity between the cognition and its object. ${ }^{18}$

Thus, Bohr's use of the concept of symbol in the broad sense can already be found in Høffding's analysis of the problem of knowledge, which in turn possesses a Kantian origin. By means of his notion of symbolic knowledge, Høffding is defending the Kantian distinction between sensibility and understanding. Both absolute idealism and naive empiricism maintain the qualitative identity of these cognitive faculties. Such identification would deny the different origins of the form and matter of our cognitions, claiming an identity where there is only an analogy. Neither is our conceptual cognition a

\footnotetext{
naturally depends quite essentially upon the kind of apparatus upon which the effect is produced. Inasmuch as the quality of our sensation gives us a report of what is peculiar to the external influence by which it is excited, it may count as a symbol of it, but not as an image. For from an image one requires some kind of alikeness with the object of which it is an image [...] But a sign need not have any kind of similarity at all with what it is the sign of. The relation between the two of them is restricted to the fact that like objects exerting an influence under like circumstances evoke like signs, and that therefore unlike signs always correspond to unlike influences." (HELMHOLTZ, 1977, p.121 - 122). For an extended discussion see Ferrari (2000). See also D'Agostino (2004).

18 Moreover, analogies and symbols play for Høffding a central role in scientific investigation. In the first place, they enable us to formulate hypothesis that guide our empirical research in realms where our knowledge is not developed yet. In new fields of investigation we presuppose that objects bear relations which are similar to the already known. In this way, analogies ground a scientific method: (HØFFDING, 1923, p.81). Secondly, by means of analogies we can unify different areas of knowledge. The particular scientific disciplines achieve a unity grounded in the similarity of the relations that they establish, even though their objects may be very different: (HØFFDING, 1923, p. 64ff.). The main influence on Høffding's ideas about the role of analogy in science comes from J.C. Maxwell and E. Mach. See Høffding (1923, p. 79). In Erkenntnis und Irrtum Mach assigns a whole chapter to the discussion of the notion of analogy, which he defines as identity of relations. The power of analogy of both to extend and to unify our knowledge is there explicitly considered by Mach, who put forward as paradigmatic the use that Maxwell makes of analogies. See Mach (1926).
} 
faint reproduction of its sensible object (as in empiricism), nor is the sensible object a confused representation of an otherwise clear, conceptual cognition (as in idealism). Rather, we may only affirm that the relations between our thoughts are identical to the relations between the sensible objects of our thoughts, i.e., in Høffding's terminology, that our knowledge is symbolic.

\section{Symbol in the Strict Sense}

In 1922, as he was writing his book on the concept of analogy (1923), Høffding consulted Bohr about this issue:

"As I mentioned to you sometime this summer, I should like to ask you about one thing in connection with your treatise about 'The Structure of the Atom and the Physical and Chemical Properties of the Elements.'

I have noticed that in most cases you use expressions indicating a relation of analogy (not of identity) between the constitution of the atoms and the actually available physical and chemical data. Examples of such expressions are 'elucidation' ( $\mathrm{p}$. $1)$, - 'explanation or rather un- derstanding' (p. 33) - 'interpret' (p. 36) - 'as the spectrum tell us and the atomic model renders understandable' ( $\mathrm{p}$. 45).

My question is whether the expression analogy would not be the expression epitomizing the terms you apply at the crucial points. All 'understanding' save pure logic - depends on analogy, and science is a strictly rational implementation of analogies between different fields of knowledge."19

At this point, Høffding is concerned with that analogy involved in our knowledge when the latter is symbolic in the broad sense. Our "physical and chemical data" do not resemble the structure of atoms, as if this structure possessed an essence independent of any theory. Therefore, there is no relation of identity between them. Rather, scientific theories stand in a relation of analogy to reality, because by means of our concepts we must only bring about order in the phenomena, the relations between phenomena so established being then identical to those expressed by the theory.

But Bohr has already realized that such a view might not be sufficient to account for the peculiarities of

\footnotetext{
${ }^{19}$ Høffding to Bohr, 20.09.22, in BCW 10, p. 511.
} 
quantum physics. In this respect, he answers:

"Here [in the theory of the atom] we are in the peculiar situation that we have fought our way to some information about the constitution of the atom, which must be considered just as certain as any of the facts of natural science. On the other hand, we meet difficulties of such a profound nature that we have no idea of the way to their solution; my personal opinion is that these difficulties are of such a kind that they hardly allow us to hope, within the world of atoms, to implement a description in space and time of the kind corresponding to our usual sensory images. Under these circumstances one must, of course, continually bear in mind that one is employing analogies and the discretion with which the areas of application of these analogies are defined in every single case is of decisive importance for making progress."20

Since, firstly, order is brought about in the phenomena by the representation of spatio-temporal images of cau- sally related objects and processes and, secondly, the mere possibility of obtaining such representations is questioned by quantum theory, a characterization of our atomic knowledge as symbolic in the broad sense would just maintain its negative meaning (in contradistinction to resembling knowledge), lacking positive content. This would be achieved only if we took into account the limited range of validity of those analogies which enable the representation of spatio-temporal images of causally related objects and processes. But in 1922 the solution to this problem could not yet be seen. In fact, such a solution is reached only in 1927 by the introduction of the point of view of complementarity. By this, a positive characterization of atomic knowledge is finally established, which is based on the notion of symbol in the strict sense. As we have seen, the necessity of the consideration of symbolism in the strict sense arises when we must represent entities which do not satisfy the conditions of the possibility of the objects of experience. In particular, the assumption of the quantum postulate in quantum theory makes us face such a situation.

Even though Høffding does not distinguish a strict from a broad sense of symbol, as Bohr does, he does consider all the necessary elements for this distinction, with the exception, of course,

\footnotetext{
${ }^{20}$ Bohr to Høffding, 22.09.22, in BCW 10, pp. 513 - 514. Already in 1913 Bohr speaks of "the most beautiful analogi [sic] between the old electrodynamics and the considerations used in my paper." BCW 2, p. 584. In December of 1922 he turns to this issue in his Nobel Lecture once again. See BCW 4, p. 482.
} 
of the quantum postulate.

To begin with, in his interpretation of Kant's theoretical philosophy, Høffding stresses the role of the law of continuity in the Kantian system. In particular, he draws the attention to the following passage of the Critique of Pure Reason (1998):

"We could easily represent the order of these four propositions (in mundo non datur hiatus, non datur saltus, non datur casus, non datur fatum) in accordance with the order of the categories, just like all the principles of transcendental origin, and show each its position, but the already practiced reader will do this for himself or easily discover the clue to it. However, they are all united simply in this, that they do not permit anything in the empirical synthesis that could violate or infringe the understanding and the continuous connection of all appearances, i.e., the unity of its concepts. For it is in this alone that the unity of experience, in which all perceptions must have their place, is possible."21

In this connection, Høffding states:
"Kant hat an dieser merkwürdigen Stelle seine ganze Erkenntnistheorie im Gesetze der Kontinuität zusammengefasst. Hätte er diesen Gesichtspunkt zu Grunde gelegt statt die logische Systematik zu befolgen, deren Konstruktion ihm zu seiner Freude gelungen war, so würde seinen Grundgedanken ihr Recht mehr geworden sein.

[...]

Der zu Grunde liegende Gedanke ist hier, dass das, was wir im Verständnisse einer Erscheinung suchen, nicht deren rein äusseres Zusammenstellen mit anderen Erscheinungen ist, sondern eine so enge und bestimmte Verbindung derselben, dass diejenige Erscheinung, welche wir zu verstehen suchen, als Fortsetzung der vorausgehende Erscheinungen dastehen und mit diesen eine kontinuierliche Reihe bilden kann. Das Zusammenhangslose und Isolierte ist uns unverständlich."( HØFFDING, 1894, p.190)

In his History of Modern Philosophy, Høffding presents the law of continuity as a condition of the possibility of experience:

21 A229/B282. 
"The law of continuity (which includes within it both the law of continuity of space and degree and the law of the causal relations of all phenomena) is valid for all phenomena, because it formulates the general conditions under which we can have real experience (as distinguished from imagination) [...] Only as the condition of experience has the law of continuity (including the causal law) validity."(1900, p.57) 22

But it is precisely the law of continuity of causality that is rejected by the quantum postulate. In this regard, Bohr states that

"the definition of every word
essentially presupposes the
continuity of phenomena and
becomes ambiguous as soon as
this presupposition no longer
applies."23

According to Bohr, the ambiguity here at stake is the impossibility of establishing a strict distinction between subject and object when the law of con- tinuity is not assumed. Since in this situation the limits of the phenomena to which the word should be applied are not well determined, this application, i.e., the definition of the word, becomes ambiguous. ${ }^{24}$ Bohr faces this problem, for example, when defining the energy difference between two stationary states. In this case, the adiabatic principle provides us with the solution:

"In this connection it may be pointed out that the principle of mechanical transformability of the stationary states allows us to overcome a fundamental difficulty which at first sight would seem to be involved in the definition of the energy difference between two stationary states which enters in relation (1) $\left[E^{\prime}-E^{\prime \prime}=h v\right]$. In fact, we have assumed that the direct transition between two such states cannot be described by ordinary mechanics, while on the other hand we possess no means of defining an energy difference between two states if there exists no possibility for a continuous mechanical connection between them. It is clear, however, that such a connection is just afforded by Ehren-

\footnotetext{
${ }^{22}$ For the specific role of continuity in Høffding's own philosophy see, for example, Høffding (1911, p. 170ff), and Høffding (1924, p.196ff).

23 BCW 6, p. 462.

24 “[T]he distinction between subject and object [is] necessary for unambiguous description."(BOHR, 1958, p. 101).
} 
fest's principle which allows us to transform mechanically the stationary states of a given system into those of another, because for the latter system we may take one in which the forces which act on the particles are very small and where we may assume that the values of the energy in all stationary states will tend to coincide."25

Under the assumption of the quantum postulate, we must accept that we cannot obtain causal and space-time images of quantum objects and processes. In this situation, one may maintain that the cognition of the law which quantum objects and processes observe is all that we need to affirm our knowledge of the quantum realm. It does not matter that we must give up using intuitive representations, because the whole cognitive value of the theory is contained in its mathematical formalism. Moreover, it may be argued that, precisely for that reason, the abandonment of the images must be considered a necessary step in the development of science.26 This would be a rationalist position.

On the contrary, from an empiricist point of view, one may maintain that the cognitive content of the theory is grounded on the regularities which constitute its empirical basis. But in this case, we should be satisfied if we can deal with these regularities, e.g., by means of protocols of experimental results, for which the use of images of quantum objects and processes is not indispensable either. ${ }^{27}$

Thus, both rationalists and empiricists may agree on the convenience of leaving images aside once the quantum postulate is assumed. Bohr, however, does not share this view. In opposition to the rationalists, Bohr demands an intuitive interpretation of the formalism, but, in contradistinction to the empiricists, he maintains that this interpretation should be carried out by means of images. More precisely, classical images should symbolically give intuitive content to the concepts of quantum objects and processes. 28

\footnotetext{
25 BCW 3, p. 75. On the limits of this solution and the further improvements carried out by Bohr see Darrigol (1992, p. 134ff).

26 This is, e.g., Cassirer's view: "The more the conceptual determination progresses, the less it proves possible to fix its results in simple particular visual images. We have to be satisfied with determination by means of laws and must forego any clarification through models." (CASSIRER, 1956, p.144).

27 The operationalism of Pauli may be considered an example of such an empiricist position: "I believe that the energy and momentum values of the stationary states are something much more real than the 'orbits'. The aim (not yet achieved) must be to deduce these and all other physically real, observable properties of the stationary states from the (integral) quantum numbers and the quantum theoretical laws. We must not, however, put the atoms in the shackles of our prejudices (of which in my opinion the assumption of the existence of electron orbits in the sense of the ordinary kinematics is an example); on the contrary, we must adapt our concepts to experience." Pauli to Bohr, 12.12.24, BCW 5, p. 429.

28 The image of an object is only the direct exhibition in space and time of the corresponding concept. It is not necessary to assume that the image resembles an independently existent object. In fact, neither Høffding nor Bohr makes such an assumption, since, as we have seen, they both consider our knowledge symbolic in the broad sense.
} 
The necessity of the use of images in the process of knowledge is a point which Høffding explicitly emphasizes:

"We cannot think without images. What we call thinking in a special and strict sense, [is] the activity by which the relation between different images is pointed out as clearly and unequivocally as possible. Every thought, be it as abstract and sublime as it may be, supposes images whose reciprocal relations it expresses." (HØFFDING 1905, p.200-201)

From a Kantian point of view, Høffding's position is justified. In fact, to cognize means, in its most general sense, to subsume a particular (that which is cognized) under a universal (the cognition), i.e., to apply the universal to the particular. For Kant, that corresponds to subsuming a sensible manifold under the concept of an object. Kant deals with the issue of this subsumption in his doctrine of schematism. The problem of schematism is precisely how a given universal is applied. The universal in question is the concept. Let us take the concept 'horse' as example. By representing to ourselves a horse we think of an object that possesses certain features (it is an animal, mammal, quadruped, with a certain colour, size and form, etc.). However, the concept 'horse' is not the mere juxtaposition of these features, because they are thought connected to each other not in an arbitrary but in a necessary way. Not any synthesis of 'animal,' 'mammal,' 'quadruped,' etc., corresponds to our concept 'horse.' Thus, the concept is not simply the consciousness of the set of a certain number of features but the consciousness of the unity of a certain synthesis of them.

In the same sense, Høffding maintains:

"The elements of the concept (e.g., colour, size and form as elements of the concept of horse) vary in the different phenomena to which it shall correspond, and the concept is therefore only possible, if the relation of the qualities is alike, though every one of them may be different. The brown colour has, e.g., the same relation to one horse as the red colour has to another horse. $\mathrm{Hu}-$ man qualities vary from man to man, but there is a certain relation between them and without this a general concept would be here impossible."(HØFFDING, 1905, p.202)

However, the representation of the unity of a certain connection of features does not provide us with the representation of the particular that possesses such features. The representation 
of the concept 'horse' is not that of a horse. How can we then link the universal concept with the representation of the particular? To answer this question the notion of schema is introduced. Kant defines schema as the "representation of a general procedure of the imagination for providing a concept with its image."29 We have just seen that the concept is the consciousness of the unity of a certain synthesis. Then the representation of the synthesis in question, as the representation of the effective process of synthesizing, is the schema of the concept. Of course, so that this synthesis can be carried out, a sensible material should be given. If this is the case, the synthesis can be done and the concept can be provided with a particular to which it is applied. This particular is an image of the concept. In our example, certain sensible material should be given (colour, size and form, etc) so that the imagination synthesizes it by means of a procedure guided by a rule whose unity is thought in the concept 'horse.'

Briefly, in an act of synthesis we should distinguish the sensible material to be synthesized (sensible manifold), the procedure by which the synthesis is carried out (schema), the representation of the unity of this procedure (concept) and the result (image). The schema is the representation of the mere act of synthesis separated from the thought of the unity that guides that synthesis and from its result. As long as the concept is the consciousness of the unity of the rule that guides the synthesis, the procedure thought in the schema generates an image of that concept as a result.

So, to cognize, i.e., to subsume a particular under a universal, means for Kant to provide a concept with its image, i.e., with an exhibition in intuition. Therefore, if we do not just consider our thinking in general, but in particular our thinking of objects, by means of which we cognize them, we must affirm that "we cannot think without images." 30

In the case of quantum objects, however, this schematic use of the concepts cannot be carried out, due to the fact that, as we have seen, the quantum postulate precludes the sensible manifold which should be subsumed under the concept of the quantum object from being given. But in that case, the demand for images is fulfilled by the symbolic use of classical images, which, in turn, are the result of the schematic application of classical concepts.

In summary, the necessity of the con-

\footnotetext{
${ }^{29}$ A140 / B179 - 180. Our emphasis.

${ }^{30}$ Kant explicitly describes our understanding as image-dependent in AA V, p. 408. Cassirer's views on quantum theory are based on the conception that symbolic knowledge does not require intuitive images. This is an important aspect of dissent between Høffding and Bohr, on the one side, and Cassirer, on the other. See Pringe (2014). Gómez points out the necessary character that Bohr assigns to the use of intuitive pictures in quantum theory in Gómez (1987, p. 20).
} 
sideration of the strict symbolic character of quantum theory is grounded on the assumptions, firstly, of the law of continuity as a condition of the possibility of experience and, secondly, of the indispensable role which images play in the process of knowledge. Since the law of continuity is rejected by the assumption of the quantum postulate, quantum objects and processes are not objects of possible experience in the Kantian sense, i.e., they cannot be represented in a causal and spatio-temporal manner. However, the knowledge provided by quantum theory must rest upon the representation of causal and spatio-temporal objects and processes in order to possess empirical content. Therefore classical images must be used to represent quantum objects indirectly in intuition. The knowledge of quantum theory will be thus symbolic in the strict sense.

The necessity for the possibility of experience of both the law of continuity and the direct exhibition in space and time of our concepts plays a central role in Bohr's account of the "epistemological lesson" of quantum theory. These views have been explicitly considered by Høffding and are moreover basic elements of Kant's critique of knowledge.

\section{Conclusions}

According to Bohr, atomic knowledge is symbolic in a twofold sense: a broad and a strict one. In the former sense, atomic knowledge is symbolic because it is not in any way a copy of a transcendent reality. In the latter sense, it is symbolic because we make use of classical concepts and images in order to represent quantum objects and processes indirectly in intuition.

The broad sense of symbol is that employed by Høffding. On the contrary, the strict sense is specifically introduced by Bohr to cope with the new situation generated by the assumption of the quantum postulate. However, the conceptual elements on which the symbolism in the strict sense is grounded are already present in Høffding's thought.

Even though only the strict sense corresponds to what Kant would call symbolic, both senses have a Kantian origin. In particular, the broad sense aims to express the rejection of the view that our concepts are to be referred to a reality in itself, in accordance with the Kantian doctrine of the insurmountable distinction between sensibility and understanding. 31

\footnotetext{
31 The project leading to this paper has received funding from the European Union's Horizon 2020 research and innovation programme under the Marie Skłodowska-Curie grant agreement No 777786. The investigation is also part of the project CONICYT/FONDECYT Regular No 1190965 and PR65/19-22446 (Comunidad de Madrid y Universidad Complutense de Madrid).
} 


\section{Bibliography}

ROSENFELD, L., RUD NIELSEN, J.,RÜDINGER, E., AASERUD, F. (eds.) Bohr's Collected Works (BCW). North-Holland, Amsterdam; American Elsevier, New York, 1972.

BOHR, N. Chemistry and The Quantum Theory of Atomic Constitution in Causality and Complementarity. The Philosophical Writings of Niels Bohr, vol. IV, Faye, J. and FolseFolse, H. (eds.), Ox Bow, Woodbridge, Connecticut, 1998

. Atomic Theory and the Description of Nature. Cambridge University Press: Cambridge, 1934.

. Atomic Physics and Human Knowledge, John Wiley \& Sons: New York, 1958.

Causality and Complementarity. The Philosophical Writings of Niels Bohr, vol. IV, Faye, J. and Folse, H. (eds.), Ox Bow, Woodbridge, Connecticut, 1998.

CASSIRER, E. Determinism and Indeterminism in Modern Physics, Benfey, T. (trans.), Yale University Press: New Haven and London, 1956.

CHRISTIANSEN, F. Heinrich Hertz's Neo-Kantian Philosophy of Science and its Development by Harald Høffding. Journal for General Philosophy of Science, 37, pp. 1-20, 2006.

D’AGOSTINO, S. The Bild Conception of Physical Theory: Helmholtz, Hertz, and Schrodinger. Physics in Perspective 6, pp. 372-389, 2004

DARRIGOL, O. From C-numbers to Q-numbers. The Classical Analogy in the History of Quantum Theory. University of California Press: Berkeley, 1992.

FAVRHOLDT, D. Niels's Bohr Philosophical Background. Historisk-filosofiske Meddeleser, 63. The Royal Danish Academy of Sciences and Letters, Copenhagen, 1992.

FAYE, J. Niels Bohr: His Heritage and Legacy.An Anti-Realist View of Quantum Mechanics. Kluwer, Dordrecht, 1991.

FERRAR,I M. Sources for the History of the Concept of Symbol from Leibniz to Cassirer in Symbol and Physical Knowledge. Springer: Berlin, 2002.

FERRARI, M., STAMATESCU, I.-O. (eds.) Symbol and Physical Knowledge. Springer: Berlin, 2002.

GÓMEZ, R. Resonancias kantianas en el pensamiento de Bohr. Revista Latinoamericana de Filosofía, XIII, pp. 3 - 23, 1987.

HELMHOlTZ, H. Epistemological Writings. The Paul Hertz \& Moritz Schlick Centenary Edition of 1921, with Notes and Commentary by the Editors, Cohen, B. (ed.), Low, M. (trans.). Reidel, Dordrecht, 1977.

HERTZ, H. The Principles of Mechanics. Dover: New York, 1956

HEISENBERG, W. The physical principles of the quantum theory. Mineola: Dover, 1949

HØFFDING, H. Die Kontinuität in philosophischen Entwicklungsgange Kants. Archiv für Geschichte der Philosophie, VII, pp. $173-192,1984$.

. A History of Modern Philosophy. A Sketch of the History of Philosophy from the Close of the Renaissance to Our Own Days, 2 vols., Macmillan, London, 1900.

. On Analogy and its Philosophical Importance. Mind, 14, pp. 199 - 209, 1905.

. Moderne Philosophen. Vorlesungen gehalten an der Universität in Kopenhagen im Herbst 1902, Reisland, Leipzig, $1905 b$.

. Der Menschliche Gedanke, Reisland, Leipzig, 1911.

. Der Begriff der Analogie. Reisland, Leipzig, 1923.

. La relativité philosophique. Alcan, Paris, 1924.

HONNER, J. The Description of Nature. Niels Bohr and the Philosophy of Quantum Physics. Clarendon Press, Oxford, 1987.

KANT, I. Kants gesammelte Schriften (AA). Königlichen Preußischen (Deutschen) Akademie der Wissenschaften: Berlin. $1902 \mathrm{ff}$.

. Critique of Pure Reason Cambridge University Press: Cambridge, 1998.

. Theoretical Philosophy after 1781. Cambridge University Press: Cambridge, 2002.

$\mathrm{MACH}$, E. Erkenntnis und Irrtum, Barth, Leipzig, 1926.

MAXWELL, J. C. The Scientific Papers. Niven, W. (ed.) (1965), Dover, New York, 1890.

PRINGE, H. Critique of the Quantum Power of Judgment. A Transcendental Foundation of Quantum Objectivity. de Gruyter, Berlín, 2007.

. Cassirer and Bohr on intuitive and symbolic knowledge in quantum physics. Theoria, 81, p. 417-429, 2014.

STOLZENBURG, K. Die Entwicklung des Bohrschen Komplementaritätsgedankes in den Jahren 1924 bis 1929, Ph.D. Thesis, Universität Stuttgart (Technische Hochschule), Stuttgart, 1977. 
Received / Recebido: 18/07/2020 Approved / Aprovado: 18/11/2020 Published / Publicado: 30/12/2020 